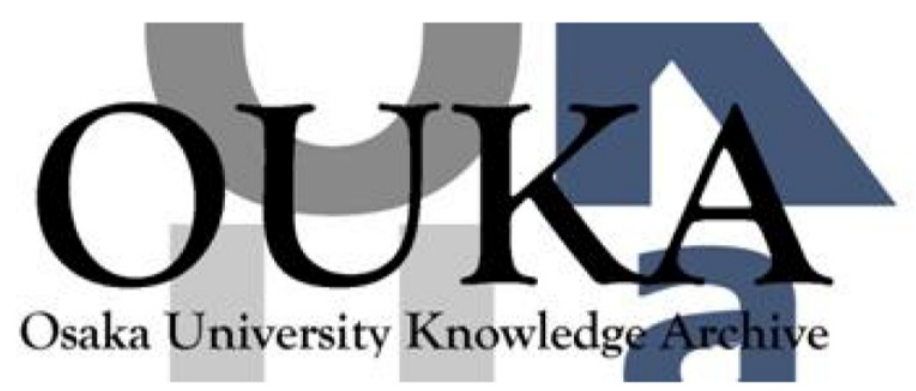

\begin{tabular}{|c|l|}
\hline Title & $\begin{array}{l}\text { Test structure for precise statistical } \\
\text { characteristics measurement of MOSFETs }\end{array}$ \\
\hline Author(s) & $\begin{array}{l}\text { Shimizu, Yoshiyuki; Nakamura, Mitsuo; Matsuoka, } \\
\text { Toshimasa et al. }\end{array}$ \\
\hline Citation & $\begin{array}{l}\text { IEEE International Conference on } \\
\text { Microelectronic Test Structures. p. 49-p. 54 }\end{array}$ \\
\hline Issue Date & $2002-04$ \\
\hline oaire:version & VoR \\
\hline URL & $\begin{array}{l}\text { https://hdl. handle. net/11094/14076 } \\
\text { c2002 IEEE. Personal use of this material is } \\
\text { permitted. However, permission to } \\
\text { reprint/republish this material for advertising } \\
\text { or promotional purposes or for creat ing new } \\
\text { collective works for resale or redistribution } \\
\text { to servers or lists, or to reuse any } \\
\text { copyrighted component of this work in other } \\
\text { works must be obtained from the IEEE.. }\end{array}$ \\
\hline rights \\
\hline Note & \begin{tabular}{l} 
\\
\hline
\end{tabular} \\
\hline
\end{tabular}

Osaka University Knowledge Archive : OUKA

https://ir. Library. osaka-u. ac. jp/

Osaka University 


\title{
3.3
}

\section{Test Structure for Precise Statistical Characteristics Measurement of MOSFETs}

\author{
Yoshiyuki Shimizu, Mitsuo Nakamura, Toshimasa Matsuoka and Kenji Taniguchi \\ Department of Electronics and Information Systems, Osaka University \\ 2-1 Yamada-oka, Suita, Osaka 565-0871, Japan
}

\begin{abstract}
A new test structure consisting of an MOSFET array and peripheral decoder circuits is proposed to study statistical variation (mismatch) in MOSFETs' characteristics. Kelvin technique was implemented in the structure to cancel parasitic resistance of metal wiring and transmission gates in such a way that any MOSFET in the array can be measured at the same bias condition. Accurate electrical measurements using the structure makes it possible to derive statistical variation of threshold voltage and transconductance of MOSFETs placed in small area.
\end{abstract}

\section{INTRODUCTION}

MOSFETs have statistical variation in device parameters such as threshold voltage $\left(V_{t h}\right)$ and transconductance $\left(g_{m}\right)[1]$. Therefore, the information of MOSFET matching for specific fabrication processes is highly required for analog circuit design because mismatch in device characteristics significantly affects the performance of MOSFET circuits, especially for high precision analog circuits. Matching evaluation requires statistical measurements using a large number of MOSFETs placed in short distance. However, a large number of MOSFETs together with measurement pads generally occupies large area. Note that both pad size and pad interval are not scaled with down-scaling because of the finite size of probing devices. For example, a $4 \mathrm{~mm} \times 4 \mathrm{~mm}$ chip with pad area of $100 \mu \mathrm{m} \times 100 \mu \mathrm{m}$ and pad interval of $150 \mu \mathrm{m}$ accommodates only 200 MOSFETs, which is not sufficient for statistical measurement.

MOSFET array structure with a decoder to select one of gate lines has been proposed to measure threshold voltage variation [2], in which the number of gate pads is significantly reduced but there still remains a large number of drain pads.

In order to further reduce the number of pads, we propose a new test structure for statistical measurement of MOSFET matching in which a decoder to select a drain line as well as a gate decoder is introduced. This drastically reduces the occupation area of an MOSFET array by cutting the number of drain pads. Key feature of the test structure is the use of Kelvin technique to avoid voltage drop in parasitic wiring and transmission gate resistance.

The test structure allows to derive statistics on MOSFET's parameters such as, $V_{t h}$ and $g_{m}$, by using an ana$\log$ IC test system which measures all the characteristics of MOSFETs in the array one after another under the same bias condition.

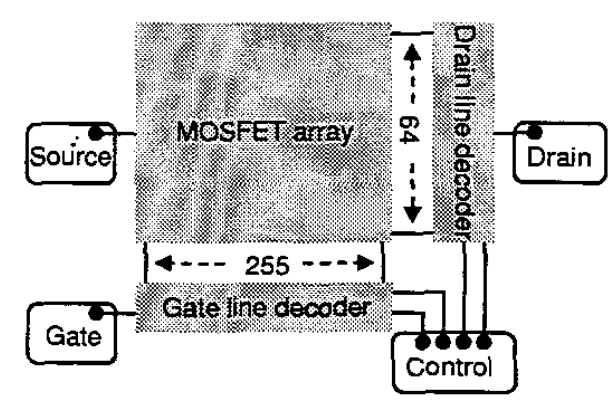

Figure 1: Block diagram of the statistical data acquisition circuit in which an array consisting of MOSFETs is included.

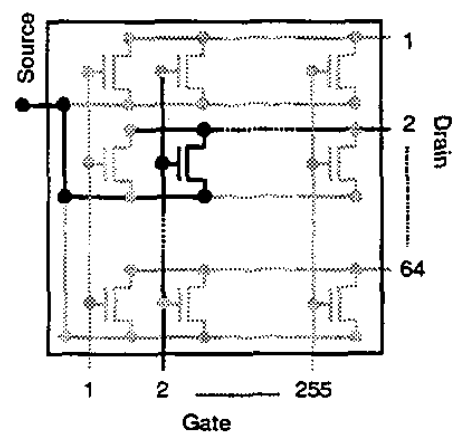

Figure 2: $255 \times 64$ MOSFETs array. Any one of MOSFETs in the array can be selected from a pair of the drain and gate lines

\section{TEST STRUCTURE}

Figure 1 shows the block diagram of the test structure for MOSFET matching measurement, which consists of an MOSFET array, gate and drain line decoders.

In the MOSFET array block shown in Fig.2, MOSFETs are placed at every nodes of a $25.5 \times 64$ array and they are connected with a common source line, where a given voltage is applied to the common gate of all the MOSFETs on a row and a given drain voltage to those on a column. Any one of the MOSFETs in the array can be selected with a pair of the gate and drain lines for the measurement at one's discretion. The gate and drain of any MOSFETs in the array are connected to their respective common pads to which external bias voltages are applied. 

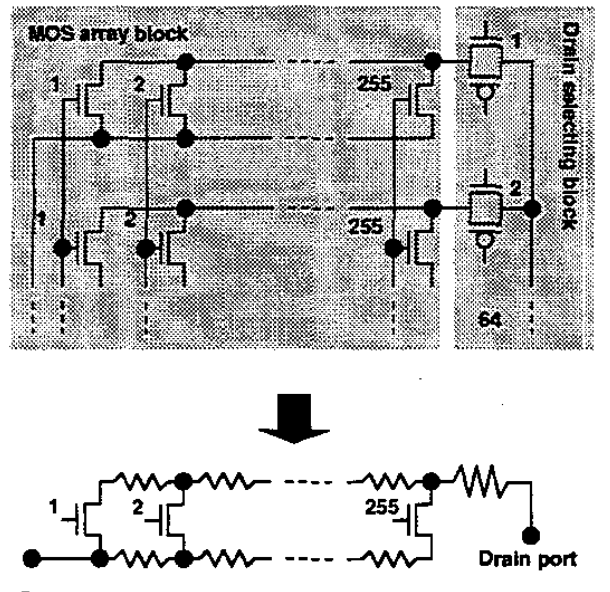

source port

Figure 3: Parasitic resistance in the circuit. Actual source and drain voltages of a selected MOSFET slightly differ from those applied to the pads due to resistance of selector switches and metal wiring.

In the test structure, there are two factors whose parasitic resistance directly affect matching measurement. One is parasitic resistance of a common drain line connected with 255 MOSFETs because of the long connecting line, typically a few $\mathrm{mm}$. The parasitic resistance depends on the location of MOSFETs measured in the array. Another is the resistance of transmission gates in the drain decoder (Figure 3).

Because of the existence of the parasitic resistance, actual drain voltage is lower than that at the external drain pad. Similarly, the source voltage of MOSFETs is higher than the external ground voltage. These parasitic effects cause significant error in mismatch measurement even at the same external bias condition.

For example, the drain current $\left(I_{D}\right)$ in linear region is given by:

$$
I_{D}=\beta\left[\left(V_{G S}-V_{t h}\right) V_{D S}-\frac{1}{2} V_{D S}^{2}\right]
$$

where $V_{D S}$ is the drain-source voltage, $V_{G S}$ the gatesource voltage and $\beta$ the current factor. Transconductance $g_{m}$, is also given by:

$$
g_{m}=\frac{\delta I_{D}}{\delta V_{G S}}=\beta V_{D S}
$$

In (2), $g_{m}$ at a given $V_{G S}$ directly depends on $V_{D S}$. Fig. 4(Conventional) shows the measured $g_{m}$ as a function of the location of the MOSFETs which clearly reflects the effect of the voltage drop in the drain line. Since 25.5 MOSFETs are connected with a common drain pad through a metal wiring, the distance from the external drain bias pad increases with decreasing the number labeled to MOSFETs $(2.55-1)$ so the effect of $V_{D S}$

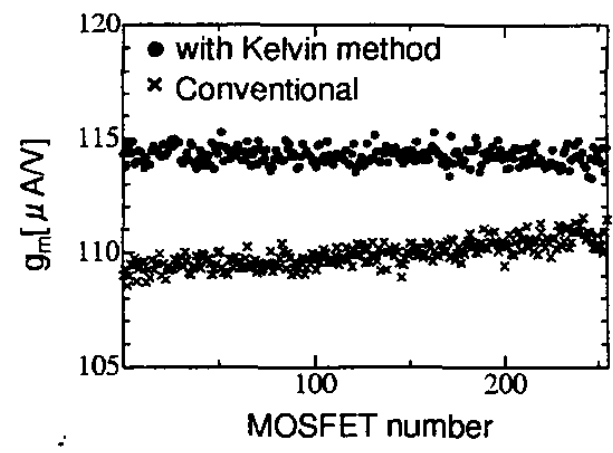

Figure 4: Systematic change of $g_{m}(X)$ due to parasitic resistance of the drain line connecting with MOSFETs with $\mathrm{W} / \mathrm{L}=10.0 \mu \mathrm{m} / 0.8 \mu \mathrm{m}$ measured at $V_{D S}=0.05 \mathrm{~V}$. The systematic change of $g_{m}$ can be avoided by using Kelvin method(O).

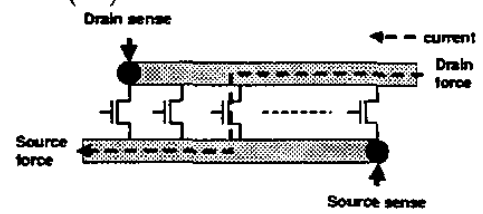

Figure 5: Technique to monitor actual drain and source voltages of a selected MOSFET. Solid circles represent the points where voltages applied to the MOSFET are monitored.

reduction due to wiring resistance is apparent: the measured $g_{m}$ decreases with the distance from the external drain pad.

To avoid the voltage drop in the drain line, we use Kelvin technique[3] for which drain and source sense points are added at the end of each line as shown in Fig. 5. Drain and source voltages of a selected MOSFET can be accurately monitored through the drain and source sense points because there exists no voltage drop due to no current between the sense points and the drain/source of a selected MOSFET. Thus, any MOSFETs in array can be measured at the same bias condition.

Solid circles in Fig. 4 represent $g_{m}$ measured by using the Kelvin method, in which there is no systematic $g_{m}$ degradation due to parasitic resistance.

Figure 6 shows the test structure including transmission gates for the drain sense, source sense and drain force ports for a selected MOSFET. The source force is common for all MOSFETs. Thus, with only a few extra circuits, $V_{t h}$ and $g_{m}$ can be evaluated accurately for all MOSFETs in the array at the same bias condition.

The parasitic resistance in the gate lines does not affect measured $V_{G S}-I_{D}$ characteristics because no DC current flows through the transmission gate connected with 64 MOSFET's' gate electrodes. 


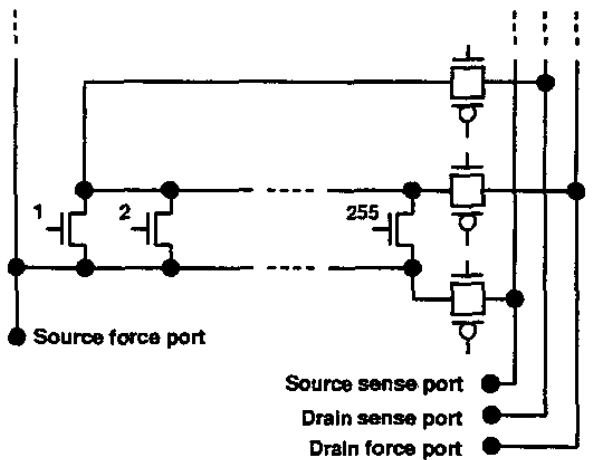

Figure 6: Auxiliary circuit to monitor actual drain and source terminal voltages by using Kelvin technique.

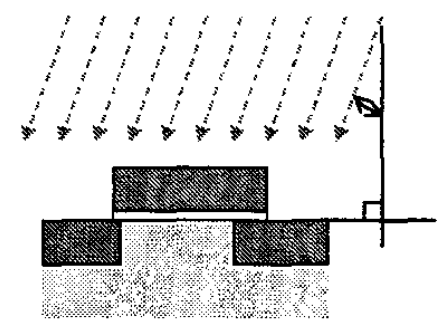

Figure 7: Asymmetric doping profile in the source and drain regions due to tilted angle ion implantation.

\section{LAYOUT OF MOSFET ARRAY}

On the physical layout of MOSFETs in the array block, the following phenomenon must be considered.

During doping process, dopant atoms are implanted into silicon substrate with an angle tilted from crystal axes to avoid ion channeling. This results in asymmetric diffusion profiles under gate electrode as show in Fig. 7 : the area of drain overlap region differs from that of source so that the exchange of drain and source would end up with different MOSFET characteristics.

To avoid this phenomenon, we placed the MOSFETs in such a way that all the drain currents flow in the same direction as show in Fig. 8.

\section{CALCULATION OF $V_{t h}$ AND $g_{m}$}

\section{LINEAR REGION}

Figure 9 shows $V_{G S}-I_{D}$ characteristics of an MOSFET operating in linear region and $g_{m}$ is derived from the maximum slope of this curve.

Threshold voltage, $V_{t h}$, is derived from the intersection of the horizontal axis and the straight line with a point where $g_{m}$ is derived.

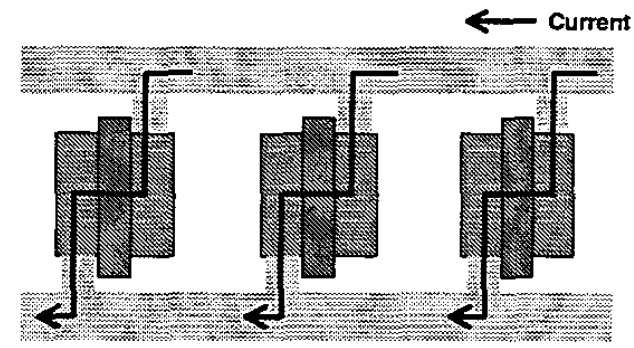

Figure 8: Layout of MOSFETs in the array block.

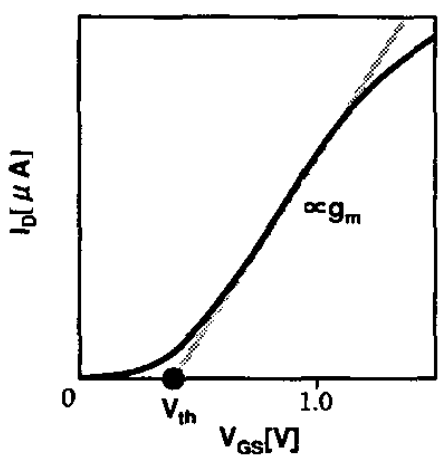

Figure 9: Both $V_{t h}$ and $g_{m}$ are derived using a line extrapolated from data measured in linear region.

\section{SATURATION REGION}

The drain current $I_{D}$ in saturation region is expressed as follows:

$$
I_{D}=\frac{\beta}{2}\left(V_{G S}-V_{t h}\right)^{2}
$$

A square root of this expression is given by:

$$
\sqrt{I_{D}}=\sqrt{\frac{\beta}{2}}\left(V_{G S}-V t h\right) .
$$

We define $V_{t h}$ as the intersection of $\sqrt{I_{D}}=0$ line and the tangent line of $V_{G S}-\sqrt{I_{D}}$ curve at the point where the slope of the curve is maximum.

The transconductance, $g_{m}$, is also extracted from the slope of $V_{G S}-\sqrt{I_{D}}$ curve, which differs from that in saturation region.

$$
\frac{\delta \sqrt{I_{D}}}{\delta V_{G S}}=\sqrt{\frac{\beta}{2}}
$$

while $g_{m}$ in saturation region is expressed as follows:

$$
g_{m}=\frac{\delta I_{D}}{\delta V_{G S}}=\beta\left(V_{G S}-V_{t h}\right)
$$

Therefore, using (4), (5) and (6), $g_{m}$ is given by:

$$
g_{m}=2 \sqrt{I_{D}} \frac{\delta \sqrt{I_{D}}}{\delta V_{G S}} .
$$




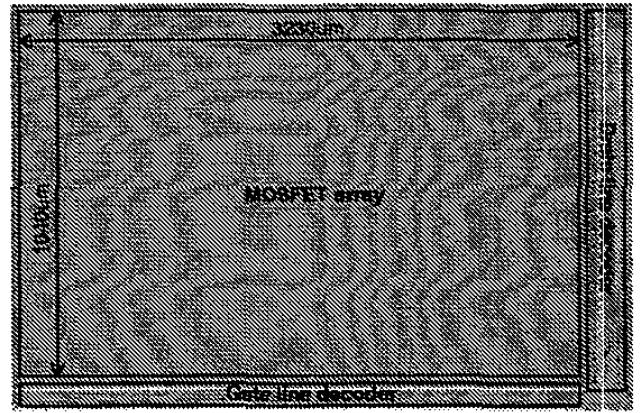

Figure 10: Photograph of the fabricated test chip.

The derivation of $g_{m}$ using ( 7 ) is little affected by leakage current on the measurement instrument. The reproducibility of $V_{t h}$ and $g_{m}$ measured was confirmed to be $\pm 0.1 \mathrm{mV}$ and $\pm 0.4 \%\left(=\sigma\left(g_{m}\right) / g_{m}, \sigma\left(g_{m}\right)\right.$ is the standard deviation of $\left.g_{m}\right)$, respectively.

\section{MEASUREMENT RESULT}

Figure 10 shows a micro-photograph of the proposed test structure. In the array, we placed 16 types of MOSFET's with different gate sizes and the number of MOSFETs is 1,020 for each size. MOSFET device parameters such as $V_{t h}$ and $g_{m}$ and their standard deviations are derived from the measured $V_{G S}-I_{D}$ characteristics of all the MOSFETs in the array. Matching characteristics of the MOSFETs in the array were measured with a mixed signal IC test system (Agilent 94000).

All the measured $V_{t h}$ and $g_{m}$ of 1,020 MOSFETs with $\mathrm{W} / \mathrm{L}=6.0 \mu \mathrm{m} / 1.0 \mu \mathrm{m}$ are shown in Fig. 11. Both $V_{t h}$ and $g_{m}$ randomly scatter around the average values. This indicates that $V_{t h}$ variation arises from a randorn event. Figure 12 shows the distributions of the measured data, which are well fitted with a Gaussian distribution[2].

Figure 13 shows the standard deviation of $V_{t h}$ as a function of the gate area. The slope of -0.51 in the log$\log$ plot indicates that the standard deviation of $V_{t h}$ is inversely proportional to the square root of the gate area, meaning that the fluctuation of $V_{t h}$ originates from the statistical variation of the number of the channel dopant as reported in Ref. [2].

Figure 14 shows deviations of $V_{t h}$ and $g_{m}$ from their averages for 1,020 MOSFETs with $\mathrm{W} / \mathrm{L}=9.0 \mu \mathrm{m} / 0.4 \mu \mathrm{m}$ measured at $V_{d s}=1.00 \mathrm{~V}$. Their distributions are shown in Fig. 15. Figure 16 shows the standard deviation of $V_{t h}$ measured at $V_{d s}=1.00 \mathrm{~V}$ as a function of the gate area.

The measurement results shown above demonstrate that the new test structure eliminates the effect of parasitic resistance in wiring and transmission gates and the variation of $V_{t h}$ and $g_{m}$ can be obtained from measurements in saturation as well as in linear regions.

\section{CONCLUSION}

We proposed a new test structure to study statistical fluctuation of MOSFETs' characteristics in a chip. This test structure consists of a MOSFET array accommodating a large number of MOSFETs in small area and peripheral decoder circuits to select one of the MOSFETS in the array which significantly reduces the number of the drain and gate pads. The test structure with $64 \times$ 255 MOSFETs subject to measurement are placed in the area of $2 \mathrm{~mm} \times 4 \mathrm{~mm}$.

The use of Kelvin technique cancels parasitic resistance in wires and transmission gates so that we accurately measure any MOSFETs in the array at the same bias condition. We demonstrated that the new test structure allows one to derive $V_{t h}$ and $g_{m}$ of any MOSFETs in the array and to analyze those variations statistically.

\section{ACKNOWLEDGMENTS}

This study was supported by VLSI Design \& Education Center (VDEC), the University of Tokyo and Japan Society for the Promotion of Science (JSPS) Research for the Future Program.

\section{References}

[1] M. J. M. Pelgrom, A. C. J. Duinmaijer and A. P. G. Welbers, "Matching Properties of MOS Transistors ,"IEEE J. Solid-State Circuits, vol. 24, no. 5, 1989.

[2] T. Mizuno, J. Okamura and A. Toriumi, "Experimental Study of Threshold Voltage Fluctuation Due to Statistical Variation of Channel Dopant Number in MOSFET's," IEEE Trans. Electron Devices, vol. 41 , no. $11,1994$.

[3] A. Hastings, "The Art of Analog Layout" (Prentice Hall, 2001), p.177. 

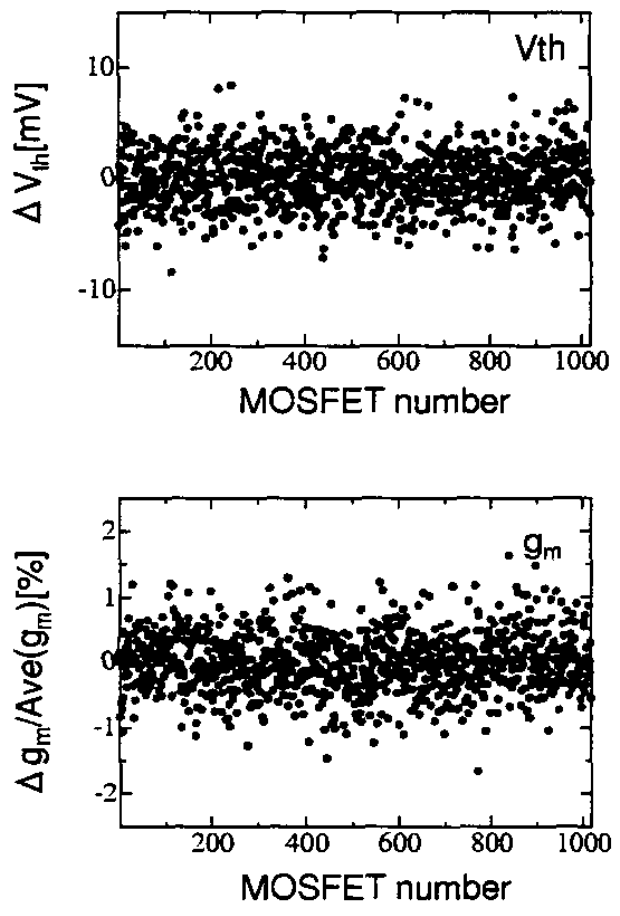

Figure 11: Fluctuation of $V_{t h}$ and $g_{m}$ from their averages derived from the data measured in linear region. The measurements were performed for 1,020 MOSFETs with $\mathrm{W} / \mathrm{L}=6.0 \mu \mathrm{m} / 1.0 \mu \mathrm{m}$ at $V_{D S}=0.05 \mathrm{~V}$.
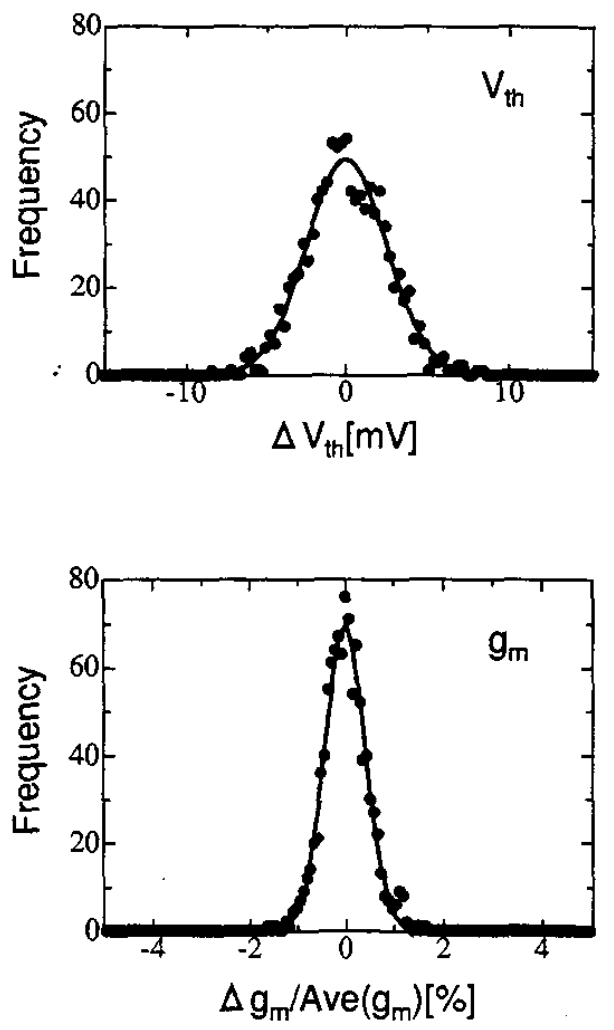

Figure 12: Frequency distribution of $V_{t h}$ and $g_{m}$ show in Fig.11.

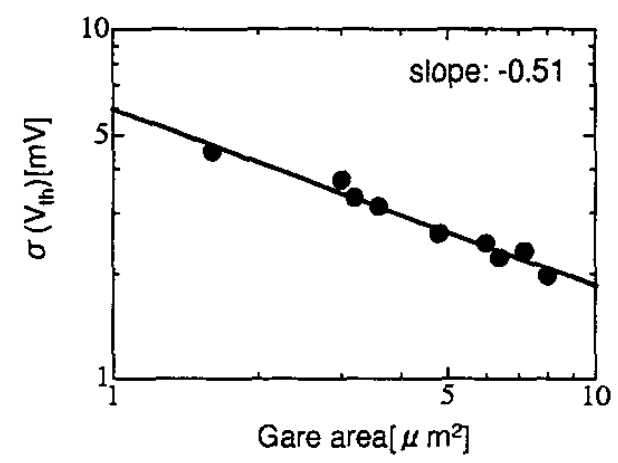

Figure 13: Standard deviation of $V_{t h}$ as a function of gate area, which were measured in the linear region. 

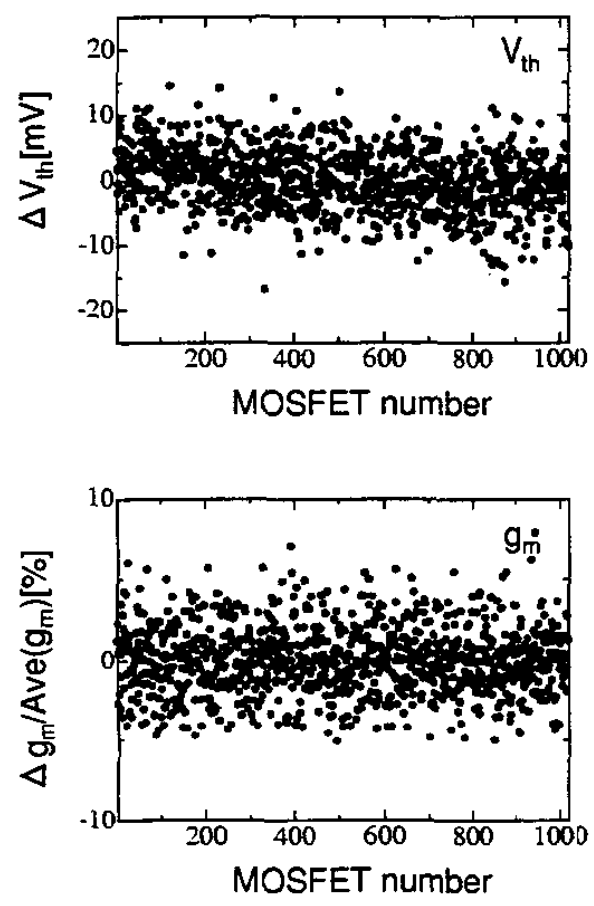

Figure 14: Fluctuation of $V_{t h}$ and $g_{m}$ in saturation region from their averages. The measurements were performed for 1,020 MOSFETs with $W / L=9.0 \mu \mathrm{m} / 0.4 \mu \mathrm{m}$ at $V_{D S}=1.0 \mathrm{~V}$.
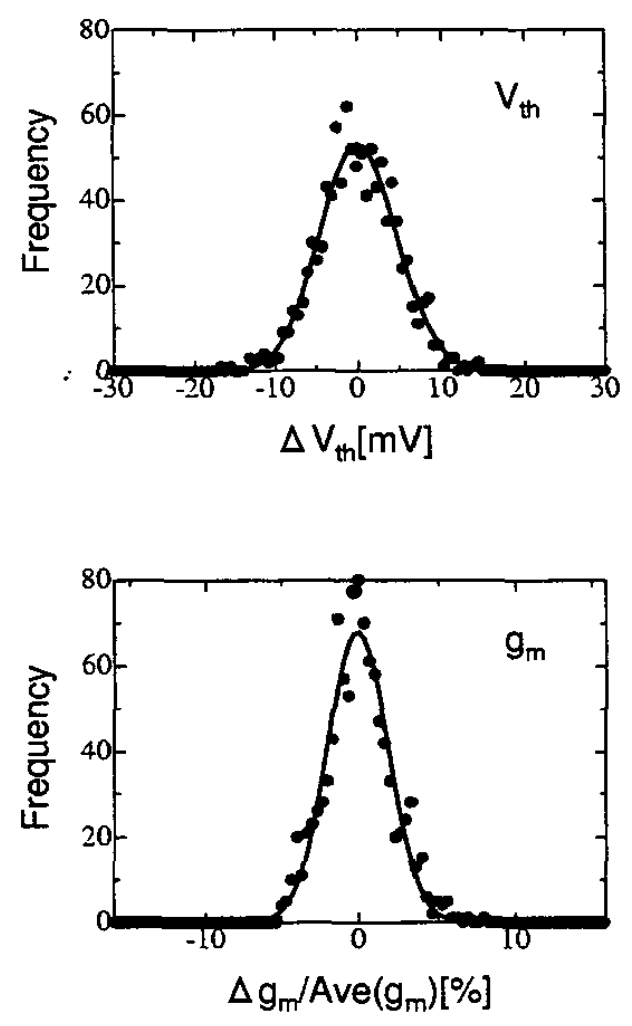

Figure 15: Statistical distribution of $V_{t h}$ and $g_{m}$ derived from the data show in Fig.14.

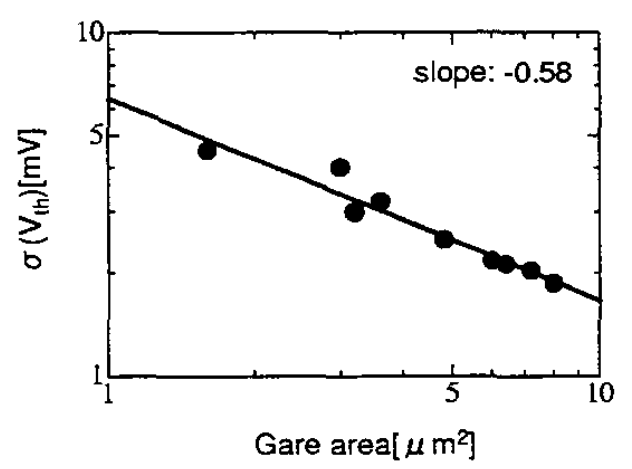

Figure 16: Standard deviation of $V_{t h}$ as a function of gate area, by measurement in saturation region. 